Research Paper

\title{
Systematic analysis of the function and prognostic value of RNA binding proteins in Colon Adenocarcinoma
}

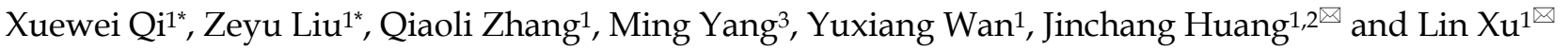 \\ 1. Third Affiliated Hospital, Beijing University of Chinese Medicine, Beijing 100029, China. \\ 2. Institute of Acupuncture and Moxibustion in Cancer Care, Beijing University of Chinese Medicine, Beijing 100029, China. \\ 3. School of Traditional Chinese Medicine, Beijing University of Chinese Medicine, Beijing 100029, China. \\ *These authors contributed equally to the work. \\ \ Corresponding authors: Jinchang Huang, E-mail: zryhhuang@163.com; Lin Xu, E-mail: 15120012660@163.com. \\ (c) The author(s). This is an open access article distributed under the terms of the Creative Commons Attribution License (https://creativecommons.org/licenses/by/4.0/). \\ See http:/ /ivyspring.com/terms for full terms and conditions.
}

Received: 2020.07.07; Accepted: 2021.02.17; Published: 2021.03.05

\begin{abstract}
Background: Abnormal expression of RNA-binding proteins (RBPs) is closely related to tumorigenesis, progression, and prognosis. This study performed systematic bioinformatic analysis of RBPs abnormally expressed in colon adenocarcinoma (COAD) using the Cancer Genome Atlas (TCGA) database to screen prognostic markers and potential therapeutic targets.

Methods: First, the gene expression data from COAD samples were used to screen out differentially expressed RBPs for functional enrichment analysis and to visualize interaction relationships. Second, RBPs that were significantly related to prognosis were screened through univariate and multivariate Cox regression analysis to construct a prognostic model. The prediction performance of the prognostic model was evaluated by survival analysis and receiver operating characteristic (ROC) curve analysis. It addition, it was verified in the test cohort. The Human Protein Atlas (HPA) online database was used to verify the expression levels of RBPs in the prognostic model.

Results: The study identified 181 differentially expressed RBPs and analyzed their interaction and functional enrichment, which were mainly related to non-coding RNA processing, ribosome biogenesis, RNA metabolic processes, RNA phosphodiester bond hydrolysis, and alternative mRNA splicing. Five RBPs related to prognosis were used to construct a prognostic model, and its predictive ability was verified by the test cohort. ROC curve analysis showed that the prognostic model had good sensitivity and specificity. Independent prognostic analysis showed that risk scores could be used as independent prognostic factors for COAD.

Conclusion: This study constructed a reliable prognostic model by analyzing COAD differentially expressed RBPs, facilitating the screening of COAD prognostic markers and therapeutic targets.
\end{abstract}

Key words: RNA-binding proteins (RBPs); colon adenocarcinoma (COAD); prognosis analysis; predictive model

\section{Introduction}

Colon cancer is one of the most common and deadly malignant tumors, presenting a serious threat to human life [1]. The incidence and morality of colon cancer have been increasing rapidly in recent years [2]. Colon adenocarcinoma (COAD) is the most common pathological type of colon cancer [3], with approximately 1.2 million new cases worldwide every year, causing 600,000 deaths [4]. Surgical resection is currently the primary treatment for localized COAD. After tumor resection, COAD is prone to relapse and metastasis. The proportion of poor prognosis in COAD patients is $25 \%$ to $40 \%$ [5, 6]. In addition, because the onset of COAD may be occult with no specific symptoms and signs, some patients are usually diagnosed at an advanced stage, which has limited therapeutic outcomes. Despite some progress in COAD diagnosis and treatment in recent years, low survival rates, high recurrence rates, and poor prognoses remain challenging [7]. Therefore, actively looking for molecular markers and therapeutic targets 
to predict the prognosis of COAD patients is of great significance for COAD treatment and improving patient prognoses.

RNA-binding protein (RBP) is an important component in post-transcriptional modification, playing an important role in tumorigenesis and tumor progression [8]. Thus far, 1,542 human genes encoding RBPs have been confirmed by experiments, accounting for approximately $7.5 \%$ of all protein coding genes [9]. RBPs interact with other proteins or RNAs to form ribonucleoprotein complexes, which regulate RNA processing, translation, exportation, and localization, thereby maintaining the stability of the intracellular environment [10]. The abnormal function of RBPs in a tumor primarily manifests in two aspects: abnormal RBP expression level, and changes in RBP activities. Related studies have shown that RBP expression in cancer tissues is significantly different from that of adjacent tissues, and is closely related to cancer patient prognosis [11-14]. Hence, systematic RBP studies enable further understanding of tumor pathogenesis and therapeutic targets, and thus are important for the identification of therapeutic targets and prognoses. Recently, there have been studies to construct cancer survival models based on RBP expression, as well as to assess the prognosis and screen therapeutic targets [15-17], but no relevant research is available for COAD.

In this study, RPBs related to COAD were obtained from the Cancer Genome Atlas (TCGA) database, and their potential functions were analyzed by identifying RBPs that were differentially expressed between tumor tissues and normal tissues. Subsequently, a prognostic prediction model was constructed to evaluate the prognosis of COAD patients, aiming to find independent prognostic biomarkers, which may better guide clinical COAD treatment.

\section{Materials and methods}

\section{Data collection and differential expression analysis}

In this study, the gene expression data and clinical information from COAD samples were obtained from the TCGA database [18] (https:// portal.gdc.cancer.gov; until May 7, 2020). RNA-SeqFPKM data were downloaded and analyzed from 398 COAD cases and 39 non-tumor tissues. Because the publishing guidelines provided by TCGA were strictly abided by, no ethical approval was required. The Limma package [19] of $R$ software was used for differential expression analysis. The Wilcoxon signedrank test was used to screen differentially expressed RBPs [9] in the tumor and normal tissues. The cut-off values were $<0.05$ for false discovery rate (FDR), and $|\log 2 \mathrm{FC}|>1$. Heat maps were generated using pheatmap software.

\section{Gene ontology and pathway enrichment analysis}

To comprehensively analyze the biological functions of these differentially expressed RBPs, the ClusterProfiler package [20] of R software was used for gene ontology (GO) [21] and Kyoto Encyclopedia of Genes and Genomes (KEGG) [22] pathway enrichment analysis. The GO terms included three categories: biological process, cellular component, and molecular function. $P<0.05$ and $q<0.05$ were used as statistically significant standards.

\section{Protein-protein interaction network construction and module selection}

To obtain the correlation of differentially expressed RBPs, these differentially expressed proteins were mapped using the Search Tool from the Retrieval of Interacting Genes/Proteins (STRING) [23] (https://string-db.org/) database, followed by utilizing Cytoscape [24] (version 3.6.1) for network visualization. The key modules of the protein-protein interaction (PPI) network were identified by the Molecular Complex Detection (MCODE) plug-in, with both MCODE score and node count number more than five [25]. $P<0.05$ was the threshold for significant difference.

\section{Prognosis model construction and verification}

Univariate Cox regression analysis was performed of RBPs in PPI, and the log-rank test was used to select RBPs related to survival. Subsequently, the entire cohort was divided into training and test cohorts. The survival-related RBPs in the training cohort were analyzed by multivariate Cox regression to construct a prognostic model and calculate a risk score in order to evaluate the prognosis of COAD patients. The formula for calculating the risk score of each sample is as follows:

$$
\text { Risk score }=\sum_{i=1}^{n}(\text { Expi } * \beta i),
$$

where $\beta$ represents the regression coefficient, and Exp represents the gene expression value.

To evaluate and verify the predictive ability of this prognostic model, the patients in the training and test cohorts were divided into low-risk and high-risk groups according to the training cohort's median risk, after which Kaplan-Meier survival analysis was performed to compare the overall survival rates of the two groups of patients. The calculation of $P$-value was performed using the log-rank test. The receiver operating characteristic (ROC) curve was prepared to evaluate the prediction accuracy of the prognosis 
model. Area under the curve (AUC) $>0.6$ was considered to be an acceptable model.

Risk score and other clinical variables (e.g., age, gender, stage, and TNM classification) together were subjected to univariate and multivariate Cox regression analyses to determine whether the risk score could be used as an independent prognostic factor. A nomogram was also prepared according to the RBPs in the prognosis model.

\section{Genetic alteration analysis and verification of expression levels}

The cBioPortal [26] (https://www.cbioportal. org/) was used to perform genetic alternation analysis on RBPs in the risk model. The Human Protein Atlas (HPA) [27] online database (http:// www.proteinatlas.org/) was used to detect the expression levels of RBPs in the prognostic model.

\section{Results}

\section{Identification of differentially expressed RBPs in COAD patients}

A flowchart of our study design is shown in

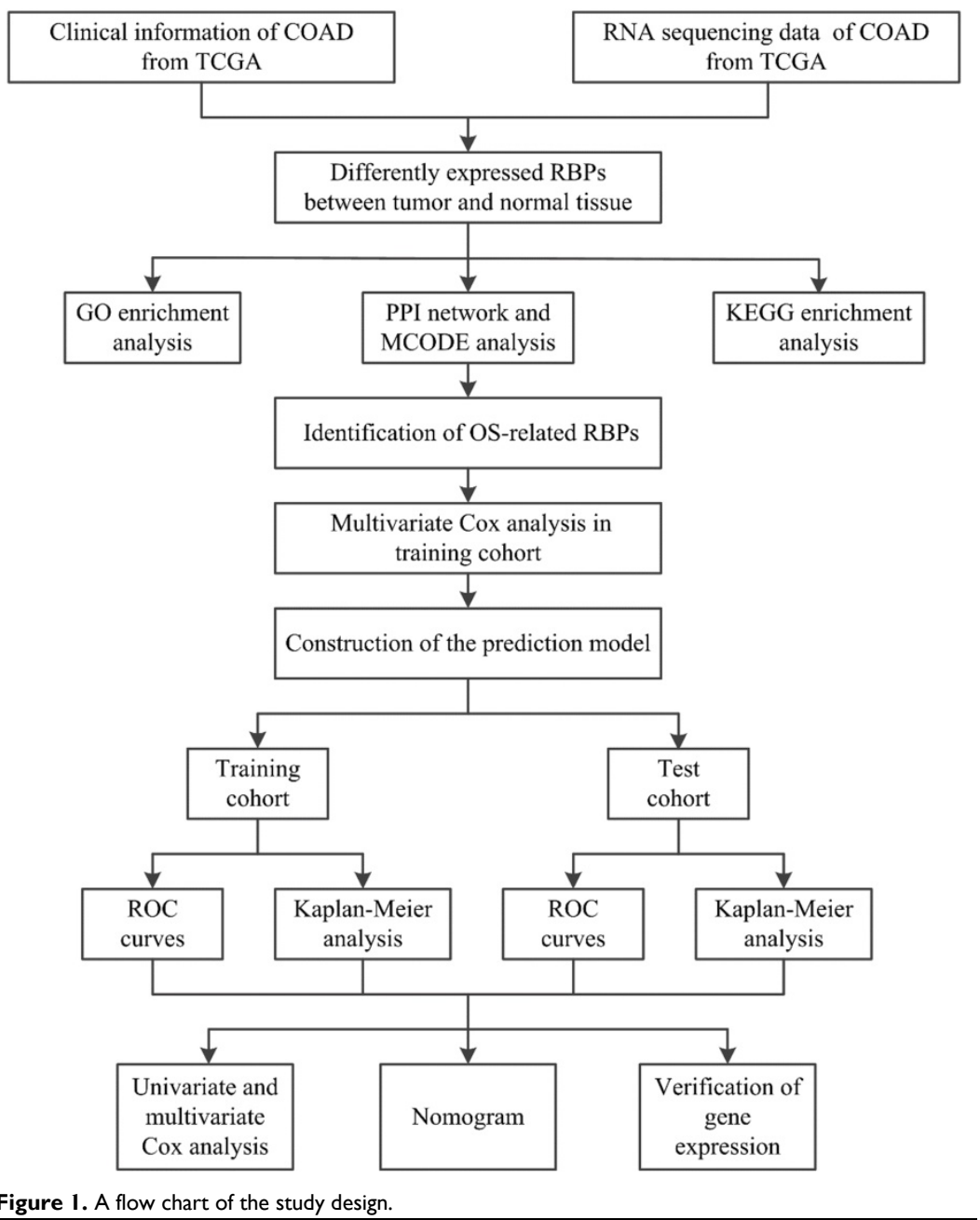

Figure 1. Clinical information and gene expression data of 398 COAD samples and 39 non-tumor tissue samples were obtained from the TCGA database, and a total of 1,375 RBPs were collected. After differential expression analysis using the Limma package of $\mathrm{R}$ software, 181 differentially expressed RBPs were identified (FDR $<0.05$ and $|\log 2 \mathrm{FC}|>1$ ), including 121 upregulated RBPs and 60 downregulated RBPs. The expression distribution of these differentially expressed RBPs is shown in Figure 2. For example, compared to non-tumor tissue, TRIM71 is upregulated and RBFOX3 is downregulated in tumor tissue. Studies have shown that TRIM71 can inhibit the expression of tumor suppressor CDKN1A/p21 and promote the proliferation of tumor cells. TRIM71 is up-regulated in hepatocellular carcinoma patients and is associated with tumor progression and poor prognosis [28].

\section{Functional enrichment analysis of the differentially expressed RBPs}

To analyze the biological functions and related signaling pathways of the differentially expressed RBPs, GO and KEGG pathway enrichment analyses were performed. The results of GO enrichment analysis showed that the upregulated differentially expressed RBPs were significantly enriched in non-coding RNA (ncRNA) processing, phosphodiester bond hydrolysis, and ribosome biogenesis. The downregulated differentially expressed RBPs were enriched in the defense response to virus, regulation of mRNA processing, regulation of translation, and regulation of mRNA metabolic processes. In terms of molecular function, the upregulated differentially expressed RBPs showing significant enrichment were involved primarily in catalytic activity acting on RNA, ribonuclease activity, and nuclease activity. The downregulated differentially expressed RBPs were significantly enriched in mRNA 3'-UTR AU-rich region binding, doublestranded RNA binding, and translation repressor activity. The results of the cellular component analysis showed that the upregulated differentially expressed RBPs were mainly enriched in cytoplasmic ribonucleoprotein granules, nucleolar components, and preribosomes, while the downregulated differentially expressed RBPs were mainly enriched in the endolysosomal 
membrane, cytoplasmic ribonucleoprotein granule, and ribonucleoprotein granule (Figure $3 \mathrm{~A}$ and $3 \mathrm{~B}$ ).

In addition, the results of the KEGG pathway enrichment analysis showed that the upregulated differentially expressed RBPs were significantly enriched in ribosome biogenesis in eukaryotes, RNA transport, mRNA surveillance pathways, and RNA degradation. The downregulated differentially expressed RBPs were significantly enriched in hepatitis C, progesterone-mediated oocyte maturation, and the toll-like receptor signaling pathway (Figure 3C and 3D).

\section{PPI network construction and module selection}

To further examine the interaction between these differentially expressed RBPs, the study utilized a PPI network using the STRING database, which contained
171 nodes and 573 edges, and was visualized using Cytoscape (Figure 4A). The medians of two topological features, degree and betweenness, were selected as the criteria, and seven pivot proteins, namely NOP56, DKC1, DDX31, DDX47, RRS1, METTL1, and PIWIL1 were obtained. Using the MCODE plug-in to process the PPI network, three key modules were selected, including module 1 , which contained 19 nodes and 161 edges(Figure 4C); module 2, which contained 8 nodes and 25 edges(Figure 4D); and module 3 , which contained 11 nodes and 28 edges (Figure 4D). The results of functional enrichment analysis showed that module 1 was mainly enriched in ncRNA processing, ribosome biogenesis, and rRNA processing; module 2 was mainly enriched in regulation of alternative mRNA splicing, alternative mRNA splicing, and regulation of mRNA splicing; and module 3 was mainly enriched in defense

A

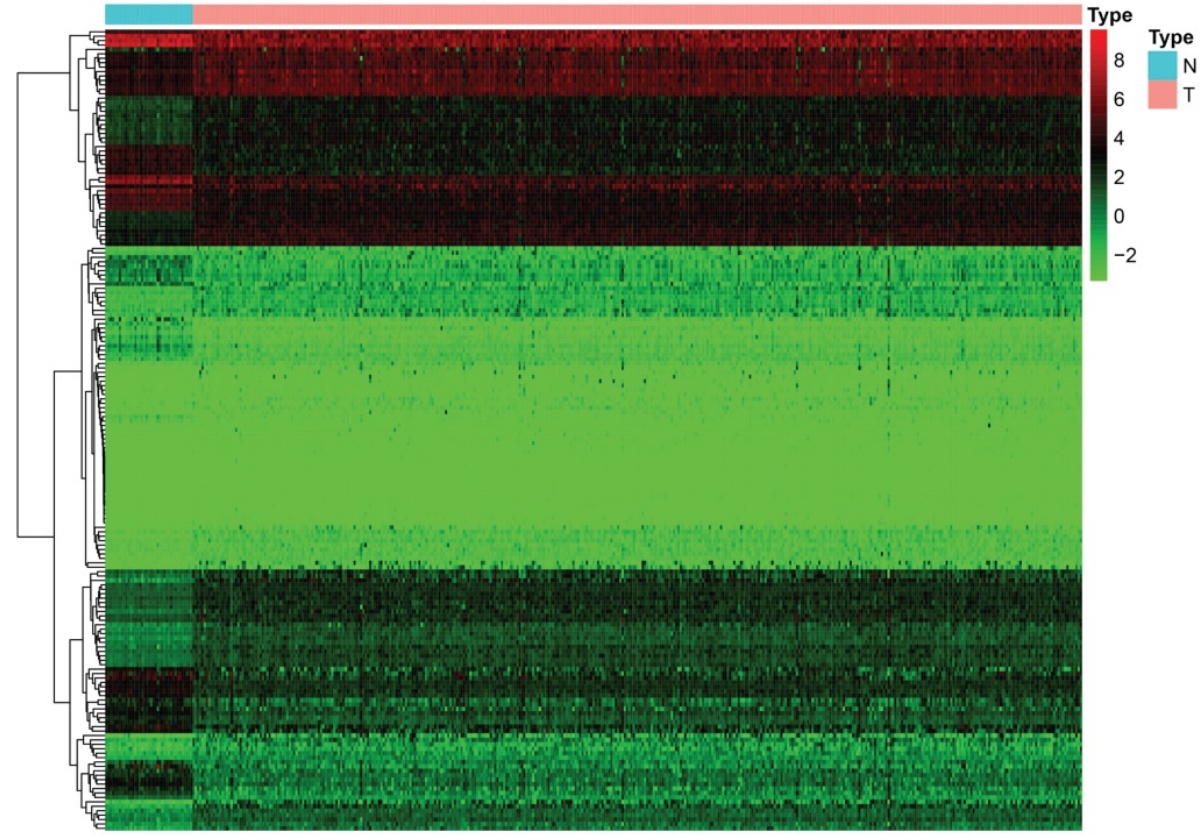

B

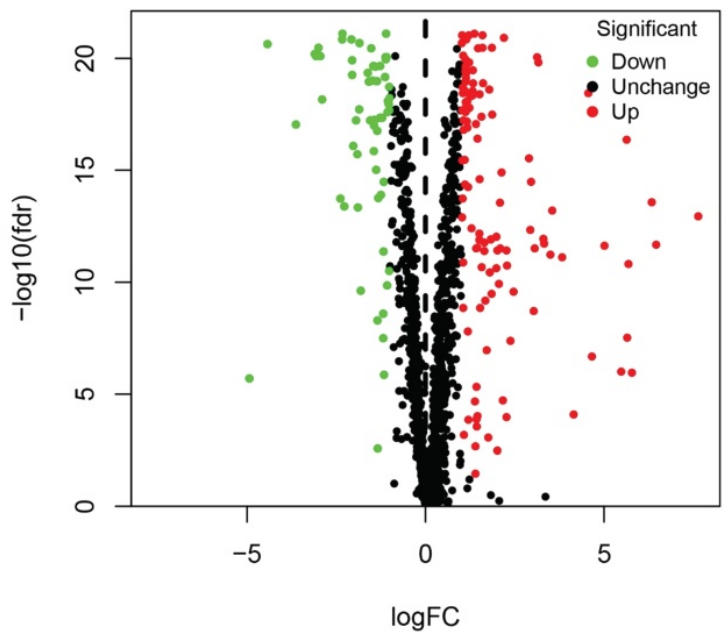

Figure 2, 181 differentially expressed RBPs in COAD, including 121 upregulated RBPs and 60 downregulated RBPs. (A) Heat map; (B) Volcano plot. 
response to virus, response to virus, and type I interferon biosynthetic process.

Table 1. 15 RBPs identified by univariate Cox regression analysis

\begin{tabular}{lllll}
\hline RBP name & Hazard ratio & Lower 95\% CI & Upper 95\% CI & $P$-value \\
\hline PNLDC1 & 1.5865 & 1.0941 & 2.3007 & 0.0149 \\
TDRD5 & 1.5915 & 1.0526 & 2.4063 & 0.0275 \\
PTRH1 & 8.2750 & 1.2506 & 54.7549 & 0.0284 \\
RBM47 & 0.5290 & 0.3271 & 0.8556 & 0.0094 \\
KHDC1L & 3.1476 & 1.6515 & 5.9989 & 0.0005 \\
LUZP4 & 433.7976 & 18.7845 & 10017.8796 & 0.0002 \\
PPARGC1A & 0.5544 & 0.3653 & 0.8414 & 0.0056 \\
PPARGC1B & 0.5061 & 0.2719 & 0.9421 & 0.0317 \\
CELF4 & 8.4467 & 2.4421 & 29.2156 & 0.0008 \\
TERT & 1.5662 & 1.0489 & 2.3388 & 0.0283 \\
POP1 & 0.5661 & 0.3232 & 0.9914 & 0.0466 \\
LRRFIP2 & 0.2851 & 0.1347 & 0.6038 & 0.0010 \\
EIF4E3 & 0.6406 & 0.4133 & 0.9930 & 0.0465 \\
LIN28B & 2.4368 & 1.3230 & 4.4882 & 0.0043 \\
TDRD7 & 0.4891 & 0.2767 & 0.8646 & 0.0139 \\
\hline
\end{tabular}

Table 2. Five prognosis-related RBPs identified by multivariate Cox regression analysis

\begin{tabular}{llllll}
\hline RBP name & Coef & Hazard ratio & Lower 95\% CI & Upper 95\% CI & $P$-value \\
\hline TDRD5 & 0.9266 & 2.5260 & 1.2790 & 4.9888 & 0.0076 \\
LUZP4 & 4.6049 & 99.9749 & 3.6527 & 2736.3292 & 0.0064 \\
\hline
\end{tabular}

\begin{tabular}{llllll}
\hline RBP name & Coef & Hazard ratio & Lower 95\% CI & Upper 95\% CI & $P$-value \\
\hline LRRFIP2 & -1.3517 & 0.2588 & 0.0807 & 0.8294 & 0.0229 \\
TDRD7 & -0.6481 & 0.5230 & 0.2226 & 1.2290 & 0.1370 \\
KHDC1L & 0.8040 & 2.2345 & 0.9579 & 5.2124 & 0.0628 \\
\hline
\end{tabular}

\section{Prognosis-related RBP selection}

A total of 171 differentially expressed RBPs were identified in PPI. To evaluate the prognostic significance of these RBPs, univariate Cox regression analysis was performed to obtain $15 \mathrm{RBPs}$ related to prognosis: PNLDC1, TDRD5, PTRH1, RBM47, KHDC1L, LUZP4, PPARGC1A, PPARGC1B, CELF4, TERT, POP1, LRRFIP2, EIF4E3, LIN28B, and TDRD7 (Table 1).

\section{Prognostic model construction and verification}

Multivariate Cox regression analysis was performed on the candidate RBPs in the training cohorts to obtain the following five RBPs for the construction of the prognostic model: TDRD5, LUZP4, LRRFIP2, TDRD7, and KHDC1L (Table 2).
A
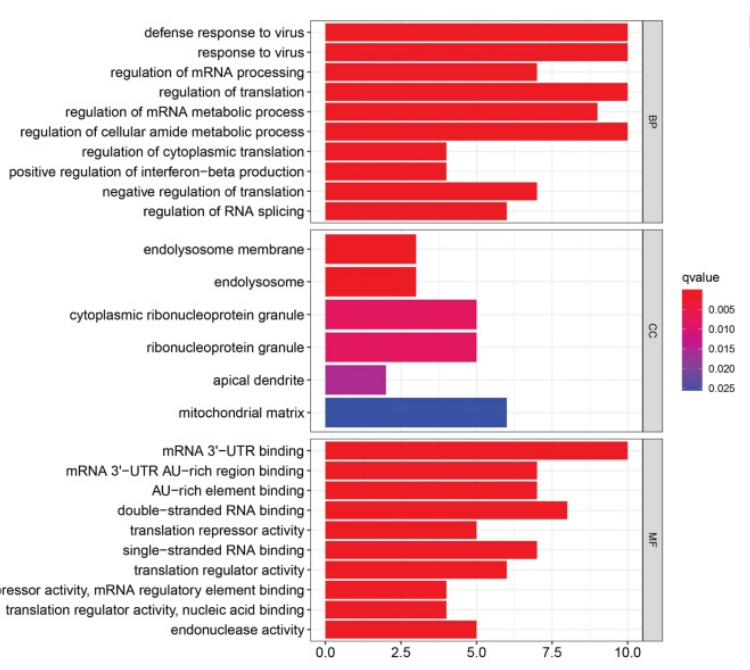

C

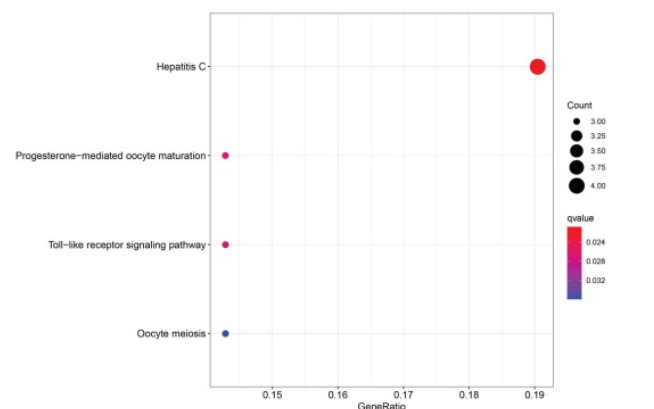

B
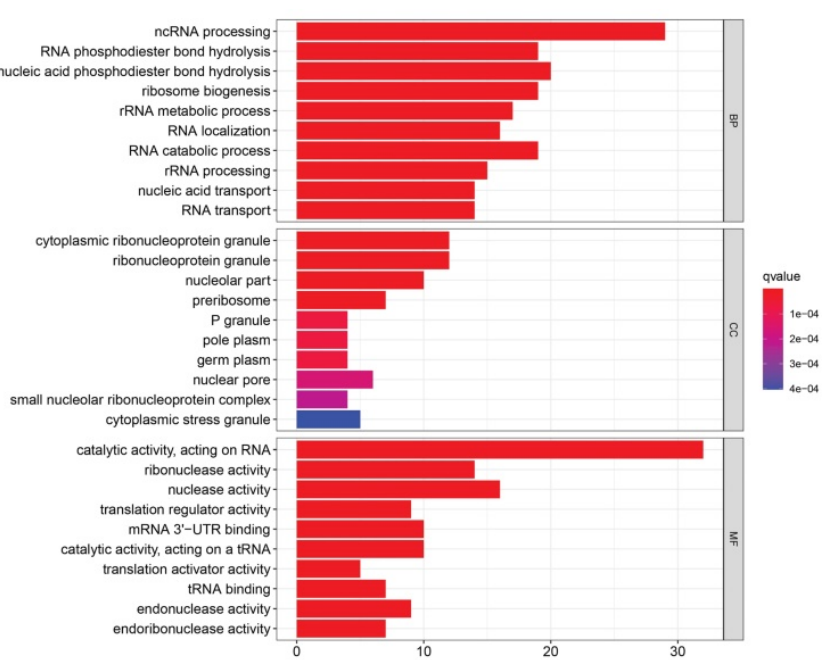

D

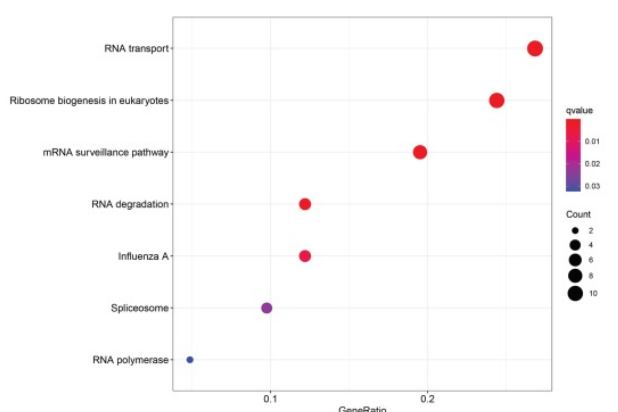

Figure 3. Functional enrichment analysis of the differentially expressed RBPs. GO enrichment analysis (BP/CC/MF) results of (A) downregulated RBPs and (B) upregulated RBPs; KEGG pathway analysis results of $(C)$ downregulated RBPs and (D) upregulated RBPs. 

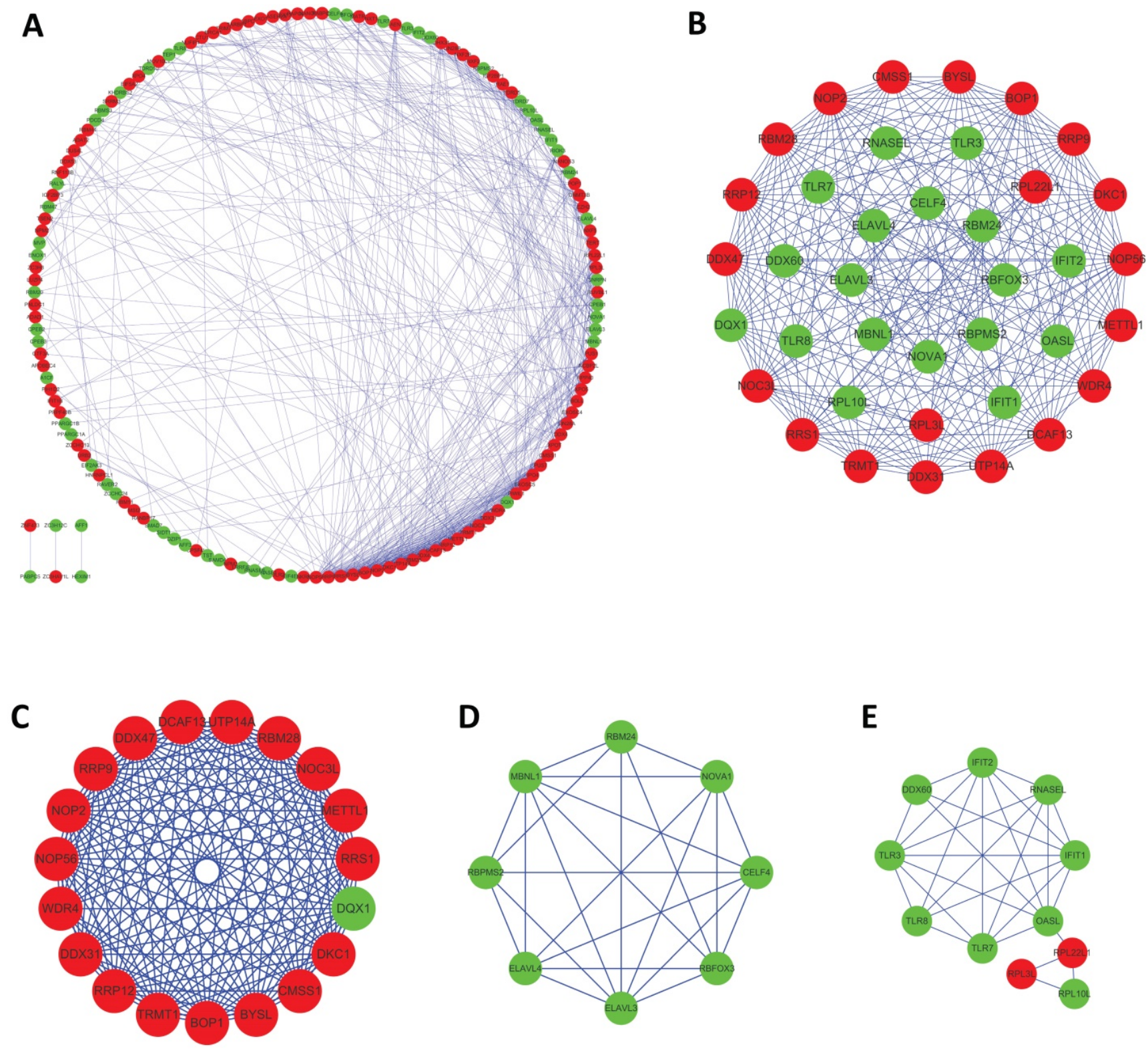

D

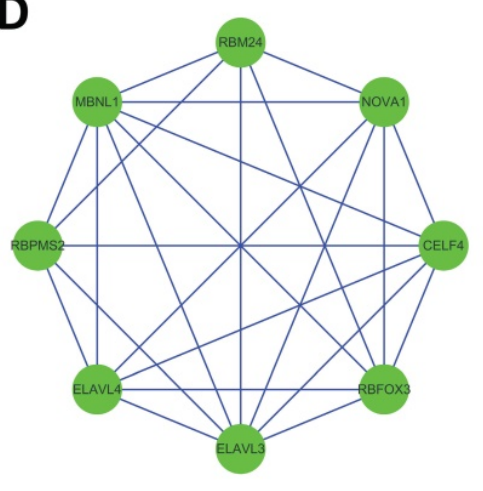

$\mathbf{E}$

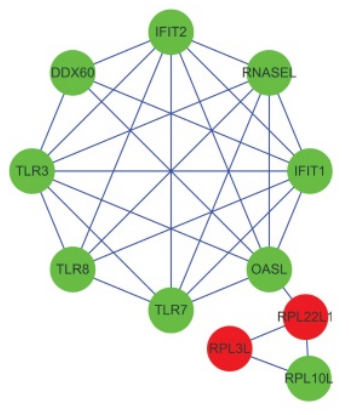

Figure 4. Protein-protein interaction (PPI) network and modules analysis. (A) PPI network of 181 differentially expressed RBPs; (B) critical module from PPI network; (C)critical module 1, (D) critical module 2 and (E)critical module 3 from PPI network. Red nodes: upregulated RBPs; green nodes: downregulated RBPs.

To evaluate the prognostic model's predicative power, 191 patients in the training cohort were divided into high-risk and low-risk groups for survival analysis based on the median risk score. The results showed that patients in the high-risk group had poorer survival rates than those in the low-risk group (Figure 5A). ROC analysis was used to test the predictive accuracy of our model, and the results showed that our model predicted the prognosis of COAD patients very well. The AUC values under the ROC curves for the one-, three-, and five-year survival rates were $0.730,0.737$, and 0.810 , respectively (Figure 5C). Figure 5 shows the risk score curves, survival status distributions, and RBPs gene expression heat map of patients in the high and low-risk groups. To further verify the accuracy of this prognostic model, survival analysis and ROC analysis were performed on a test cohort consisting of 188 patients, and the results showed that the survival rates of the high and low-risk groups were significantly different. The AUC values under the ROC curves for the one-, three-, and five-year survival rate were $0.680,0.682$, and 0.661 , respectively, suggesting that our prognostic model had good sensitivity and specificity (Figure 5).

In addition, univariate and multivariate Cox regression analyses were used to evaluate the risk scores of our prognostic model and prognostic values of other clinical features. The univariate Cox regression analysis suggested that stage, TNM classification, and risk score were associated with 
COAD prognosis (Figure 6A). The multivariate Cox regression analysis suggested that risk score may be an independent risk factor (Figure 6B).

\section{Construction of a nomogram based on RBPs in the prognostic model}

To better predict the survival time of COAD patients, a nomogram based on the gene expression of RBPs in the prognosis model was constructed (Figure
7). The expression of each RBP in the prognostic model corresponded to a point. All points were added to calculate the total points of each patient. The one-, two-, and three-year survival rates of the patients were predicted by drawing perpendicular lines between the axis of total points and each prognostic axis.
A
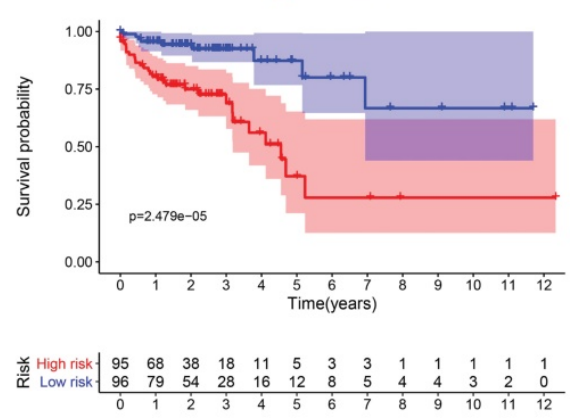

C

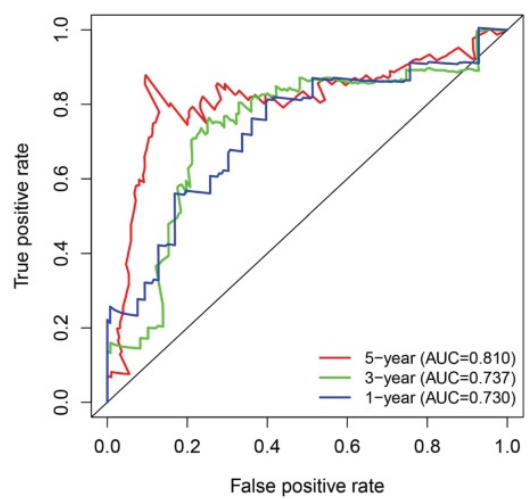

E

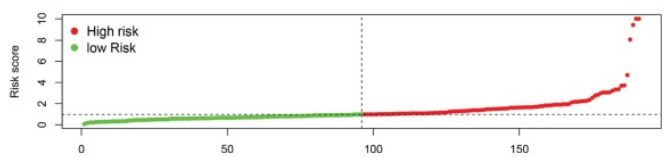

G

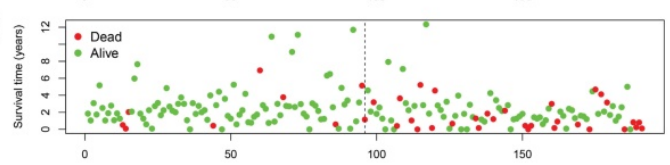

I

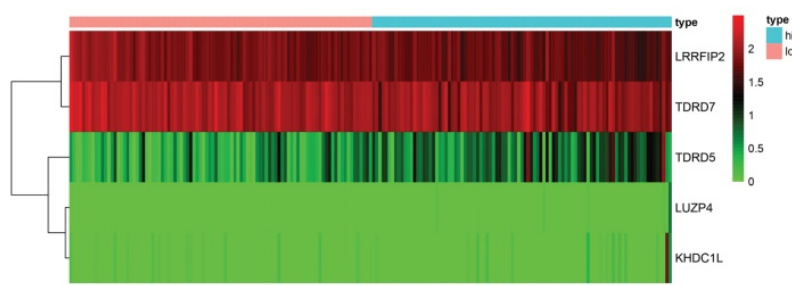

B
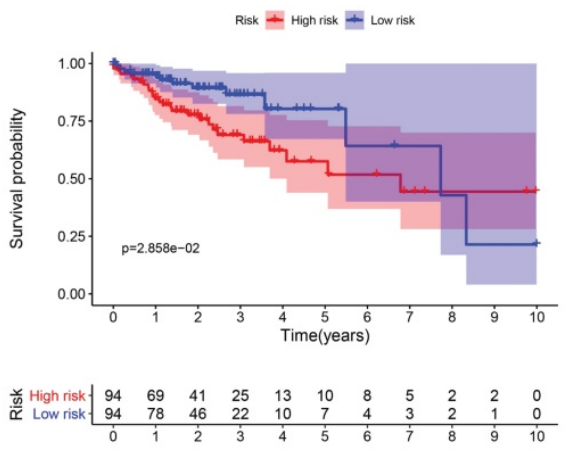

D

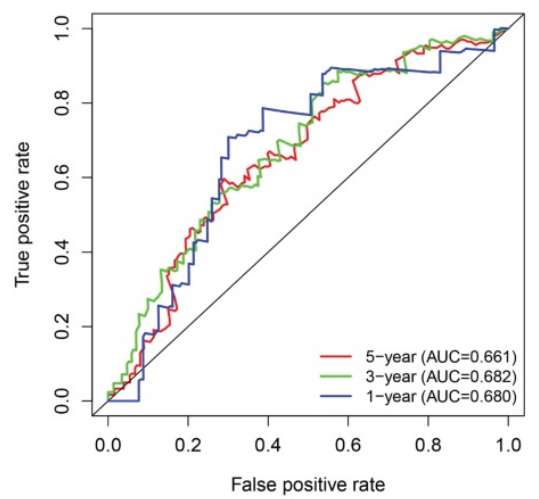

F

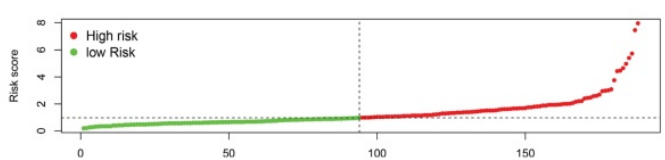

H
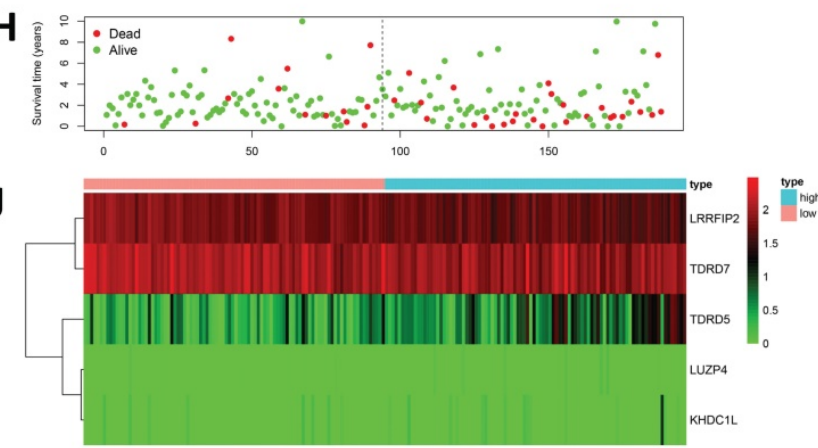

Figure 5. Risk score analysis of prognostic model in TCGA cohort. (A) Kaplan-Meier curves and (C) ROC analysis of the prognostic model in TCGA training cohort; (B) Kaplan-Meier curves and (D) ROC analysis of the prognostic model in TCGA test cohort; (E) risk score curves, (G) survival status and (I) heat map of COAD patients in TCGA training cohort and training cohort.; $(\mathrm{F})$ risk score curves, $(\mathrm{H})$ survival status and $(\mathrm{J})$ heat map of COAD patients in TCGA test cohort. 


$\begin{array}{crr} & \text { pvalue } & \text { Hazard ratio } \\ \text { age } & 0.006 & 1.032(1.009-1.055) \\ \text { gender } & 0.996 & 0.999(0.614-1.624) \\ \text { stage } & <0.001 & 2.457(1.850-3.263) \\ \text { T } & <0.001 & 3.229(1.972-5.288) \\ \text { M } & <0.001 & 5.078(3.062-8.421) \\ \text { N } & <0.001 & 2.142(1.608-2.853) \\ \text { riskScore } & <0.001 & 1.094(1.059-1.130)\end{array}$
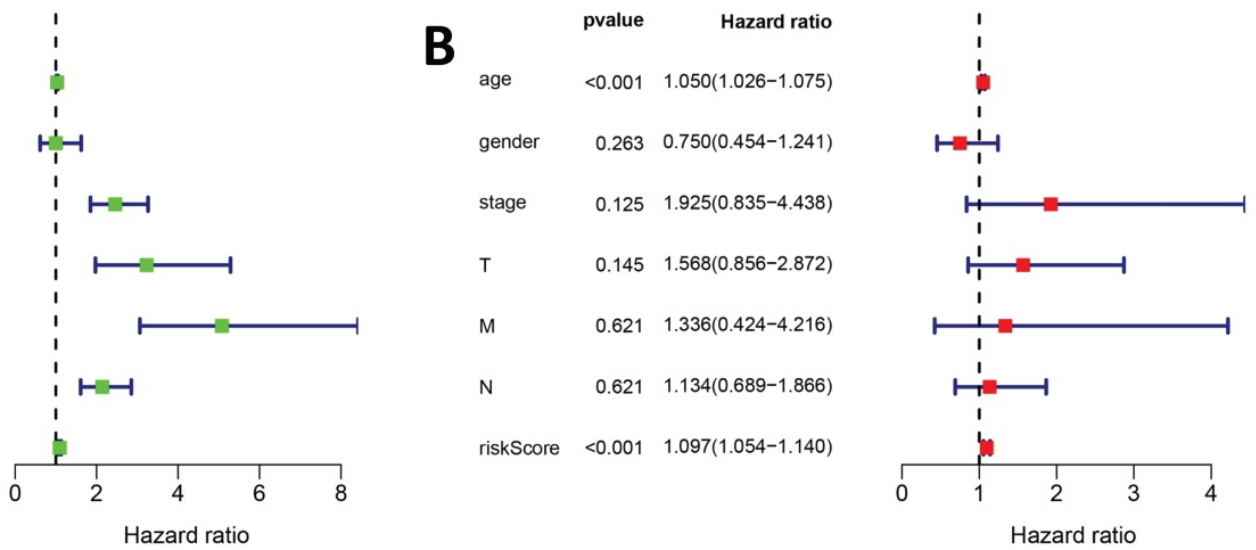

Figure 6. The Cox regression analysis for evaluating the independent prognostic value of the risk score of the prediction model. The (A) univariate and (B) multivariate Cox regression analysis of age, gender, stage, TNM classification and risk score.

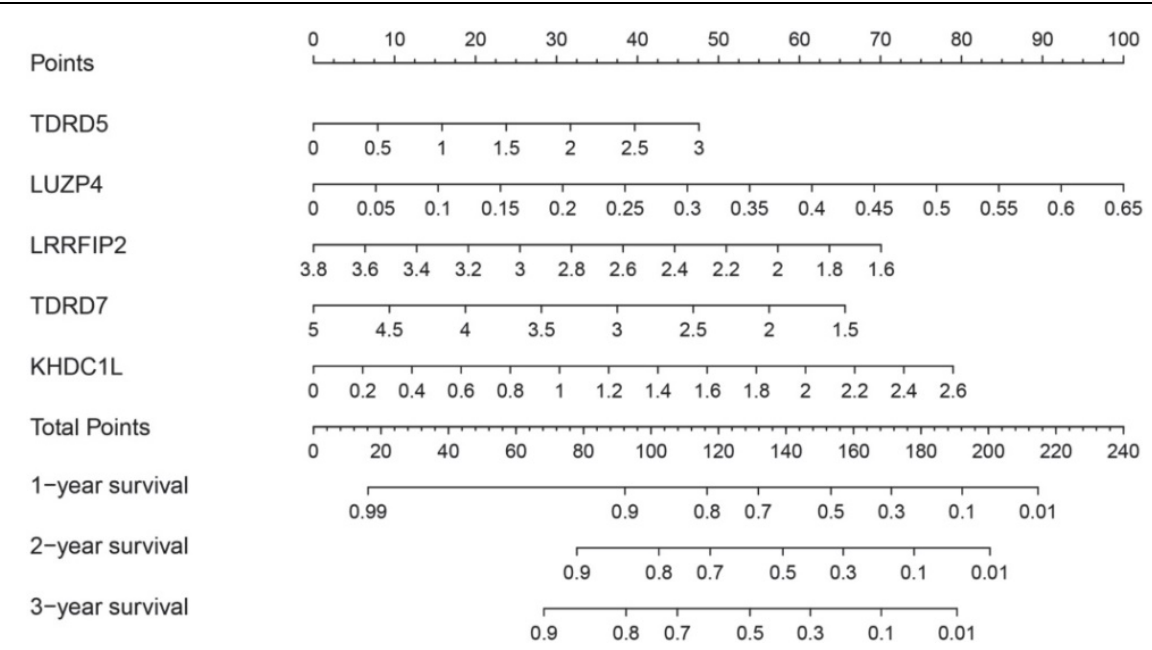

Figure 7. Nomogram of predicting 1-, 2-, and 3-years OS of COAD patients in the TCGA cohort.

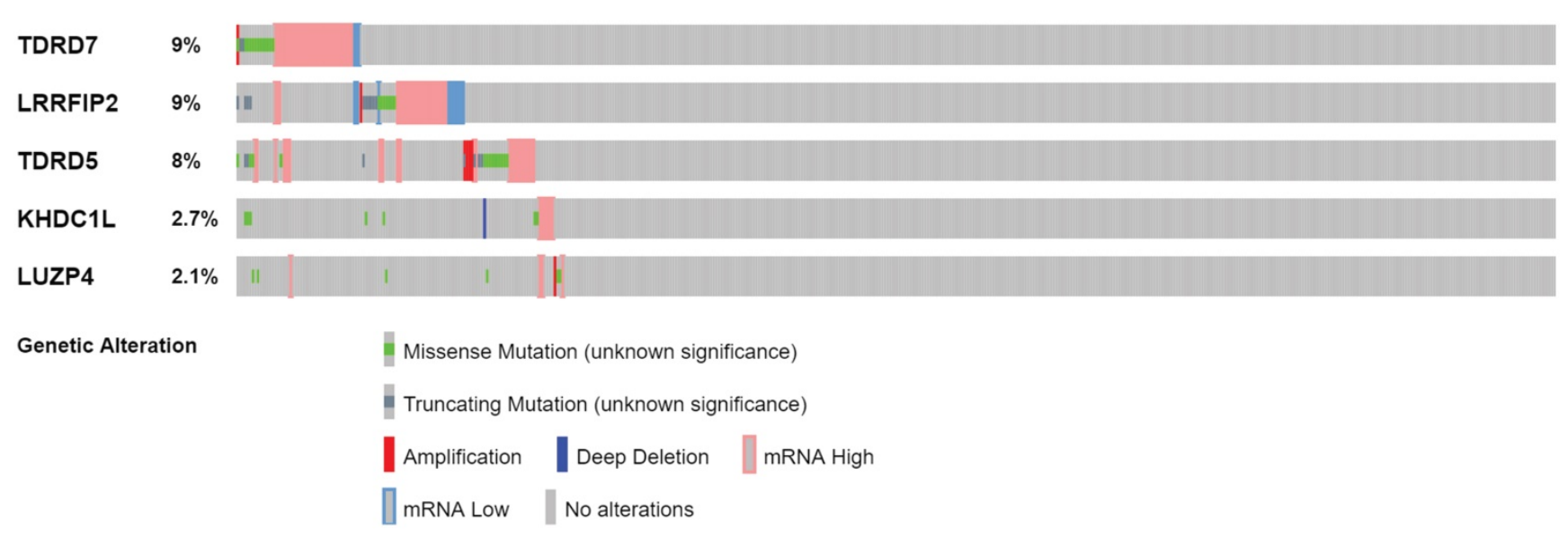

Figure 8. Genetic alteration analysis of 5 prognosis-related RBPs in COAD patients.

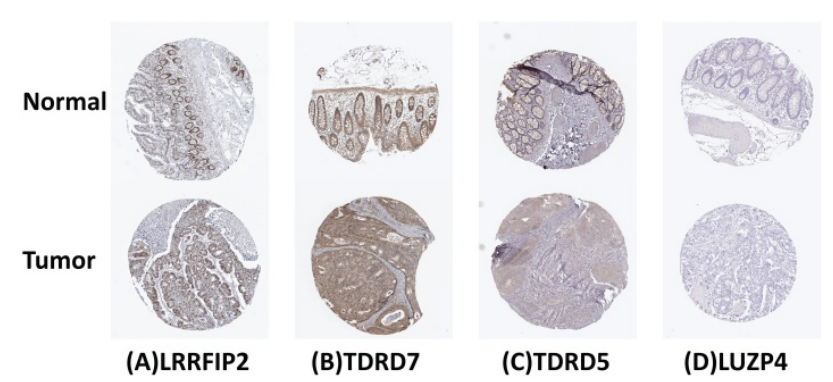

Figure 9. Verification of 4 prognosis-related RBPs expression in COAD and normal colon tissue using the HPA database. (A) LRRFIP2, (B) TDRD7, (C) TDRD5, (D) LUZP4. 


\section{Genetic alteration analysis and expression level verification}

The cBioPortal was used to perform genetic alteration analysis on five genes related to prognosis, TDRD5, LUZP4, LRRFIP2, TDRD7, and KHDC1, and the results showed that 130 of 524 COAD samples $(25 \%)$ had undergone alternation among the listed genes. The alteration was dominated by mRNA upregulation (Figure 8). To verify the differences expression for these five genes, the immunohistochemical results of the five genes expressed in COAD were obtained from the HPA database. LRRFIP2 and TDRD7 expression in COAD tissues were significantly higher than those in the normal colon tissues. In addition, TDRD5 expression was weakly positive in COAD tissues and was negative in normal colon tissues. LUZP4 expression was negative in both normal colon and COAD tissues (Figure 9).

\section{Discussion}

RBPs are an important component of gene expression pathways in eukaryotes and are key regulators of post-transcriptional processes that mediate RNA maturation, transport, localization, and translation [29]. The different functions of RBPs in post-transcriptional gene regulation are crucial for cell differentiation and proliferation. Therefore, abnormal expression of RBPs leads to the occurrence of various human diseases, including cancer. RBPs are well recognized to play a key role in tumorigenesis and tumor progression. However, many of their specific roles in cancer biology have not been discovered. Therefore, analysis of the differentially expressed RBP network and related functions is helpful to promote in-depth understanding of their role of tumor biology, possibly revealing new targets for cancer treatment [30]. COAD is one of the most common gastrointestinal malignancies, with a low survival rate, high recurrence rate, and poor prognosis. Therefore, screening of prognostic biomarkers and new therapeutic targets has great significance for early therapeutic intervention and prognosis for COAD.

This study analyzed RBPs in 398 COAD and 39 non-tumor tissue samples using TCGA to identify 181 differentially expressed RBPs, including 121 upregulated and 60 downregulated RBPs. The biological functions related to the differentially expressed RBPs were subsequently and systematically analyzed to construct a PPI network. Univariate and multivariate Cox regression analyses, survival analysis, and ROC analysis were used to further explore the biological functions and clinical significance of the differentially expressed RBPs. This study built a prognostic risk model based on five
RBPs that were significantly associated with prognosis, and performed cohort verification using the TCGA database. This model can be used to facilitate prognostic analysis and treatment for COAD patients and to develop new biomarkers.

The results of our enrichment analyses of the biological functions of differentially expressed RBPs and related signaling pathways showed that differentially expressed RBPs were enriched on multiple GO terms. In terms of biological process, upregulated differentially expressed RBPs are significant enriched in ncRNA processing, RNA phosphodiester bond hydrolysis, and ribosome biogenesis. Downregulated differentially expressed RBPs are significantly enriched in defense response to virus, regulation of mRNA processing, regulation of translation and regulation of mRNA metabolic process. The ncRNA has been the focus of cancer research [31]. The abnormal regulation of lncRNA is related to metastasis and recurrence of many cancers [32]. Studies have reported that the expression of ZEB1-AS1 in COAD is significantly upregulated and is related to poor prognosis. The miR-455-3p/PAK2 axis can promote the malignant progression of COAD [33]. Ribosomal biogenesis plays an important role in tumorigenesis and tumor progression. Both the upregulation of ribosomal biogenesis and internal dysfunction lead to increased genomic stability and decreased activity of tumor suppressor gene p53, increasing the risk of cancer [34]. In terms of molecular function, upregulated differentially expressed RBPs are mainly enriched in scatalytic activity acting on RNA, ribonuclease activity, and nuclease activity. Downregulated differentially expressed RBPs are mainly enriched in mRNA 3'-UTR AU-rich region binding, double-stranded RNA binding, and translation repressor activity. RBPs can bind to various types of RNA and regulate the activity of various enzymes. Ribonuclease can inhibit the proliferation of tumor cells by catalyzing the cleavage of phosphodiester bonds in various single-stranded RNAs [35]. In terms of cellular component, upregulated differentially expressed RBPs are mainly enriched in cytoplasmic ribonucleoprotein granule, nucleolar part, and preribosome. Downregulated differentially expressed RBPs are mainly enriched in endolysosome membrane, cytoplasmic ribonucleoprotein granule, and ribonucleoprotein granule. In recent years, mutations in ribosomal protein genes have been found in different types of cancer, such as ribosomal protein S20 (RPS20) gene mutations in colorectal cancer [36]. Studies have also shown that ribosomal protein S3 (RPS3) is highly expressed in colon cancer. Knockout of RPS3 can significantly inhibit the proliferation and migration of colon cancer 
Caco-2 cells, up-regulate the expression of p53 protein, and increase tumor cell apoptosis [37]. The KEGG pathway enrichment analysis showed that abnormally expressed RBPs regulated tumorigenesis and COAD progression by affecting ribosome biogenesis in eukaryotes, as well as RNA transport, mRNA surveillance pathways, and RNA degradation. In a previous study that included expression of RBPs in 16 different types of cancers [38], the results showed that RBPs with functions in RNA splicing, translation, transcription termination, RNA localization and transport, RNA surveillance and degradation, RNA modification, ribosome, transfer RNA (tRNA), and other functions were significantly upregulated in COAD tissues, which were consistent with our findings.

In addition, a PPI network of differentially expressed RBPs was constructed to obtain seven hub proteins, including NOP56, DKC1, DDX31, DDX47, RRS1, METTL1, and PIWIL1. Enhanced ribosomal biogenesis and increased protein synthesis are important features of cancer cell proliferation [39]. NOP56 is a core protein member of box C/D small nucleolar RNPs (snoRNPs) and plays an important role in ribosome biosynthesis [40]. DKC1 encodes dyskerin protein and is involved in rRNA processing, folding, and modification. Multiple studies have shown that DKC1 is upregulated in colorectal cancer, prostate cancer, breast cancer, and other cancers, indicating that DKC1 upregulation may be a common feature of invasive cancer [42, 42]. DDX31 is a nucleolar protein, and DDX31 overexpression is related to p53 mutation and estimated glomerular filtration rate (eGFR), which promotes the invasion and migration of bladder cancer [43]. DDX31 interacts with and co-localizes with the NPM1 protein in the nucleolus of kidney cancer cells, regulating the p53 pathway and rRNA gene transcription, thereby playing a key role in tumorigenesis and progression of kidney cancer [44]. DEAD-box RNA helicases also play important roles in ribosome biogenesis, RNA processing and folding, RNP remodeling, RNA nuclear export, the regulation of RNA translation and transcription, and other processes that are closely related to tumorigenesis. DDX47 is an important helicase that is mainly involved in pre-rRNA processing [45]. The main function of RRS1 is to participate in ribosome biogenesis. A previous study has shown that RRS1 expression in colorectal cancer is higher than in the tumor-adjacent normal tissues. Downregulation of RRS1 induces G2/M cell cycle arrest, apoptosis, and angiogenesis, thereby inhibiting the proliferation of colorectal cancer cells [46]. In addition, RRS1 overexpression is related to the tumorigenesis and progression of several tumor types, such as gastric cancer, hepatocellular carcinoma, and cervical cancer [47-49]. RNA modification is related to the tumorigenesis of various cancers, and the METTL family is a key modifier of tRNA and rRNA. METTL1 mainly regulates the modification of N7-methylguanosine (m7G) and plays an important role in the progression of tumors, such as colon cancer, liver cancer, and lung cancer [50-52]. PIWIL1 is an important member of the Argonaute protein family that is closely related to the biological behaviors of tumor cell proliferation, apoptosis, adhesion, metastasis, and chemotherapy resistance [53]. Studies have shown that the expression of PIWIL1 in colorectal cancer is significantly higher than that of the tumor-adjacent tissues, and that its expression is closely related to the degree of tumor differentiation, depth of tumor invasion, and TNM stage, thereby promoting the growth, proliferation, and invasion of colorectal cancer [54,55]. By analyzing the main modules in the PPI network, we found that these modules were primarily related to ncRNA processing, ribosome biogenesis, regulation of alternative mRNA splicing, and defense response to virus. RBPs-mediated post-transcriptional regulation is one of the important regulatory mechanisms of lncRNA, which mainly regulates lncRNA stability, transport and localization, and plays an important role in the occurrence and development of cancer [56]. For example, heterogeneous nuclear ribonucleoprotein $\mathrm{K}$ (hnRNPK), a RBP that plays a role in the nuclear accumulation of lncRNAs, is upregulated in colorectal cancer, gastric cancer and other cancers, and is associated with poor prognosis[57,58]. RBP can participate in the biogenesis of ribosomes, and the abnormality of ribosomal biogenesis is closely related to the occurrence and development of cancers [59]. RBPs regulate gene expression through post-transcriptional regulation, such as alternative mRNA splicing and microRNA processing. Abnormal mRNA splicing is a common driving factor in the occurrence and development of cancer, affecting the phenotype of cancer cells, including proliferation, apoptosis, invasion and transfer [60]. In colorectal cancer, SRSF3 interacts with the small splice-regulating protein SRSP, mediating selective splicing of SP4 to produce cancerous SP4 subtypes, which leads to tumor occurrence and metastasis [61].

Subsequently, 15 RBPs related to prognosis were obtained based on univariate Cox regression, and five RBPs (TDRD5, LUZP4, LRRFIP2, TDRD7, and KHDC1L) obtained from the multivariate Cox regression analysis using the TCGA training cohort were used for the construction of a prognostic risk model. TDRD5 could be combined with PIWI-interacting RNA (piRNA) precursors, and plays 
a key role in piRNA biogenesis [62]. A previous study has shown that TDRD5 expression is upregulated in hepatocellular carcinoma, which has value for determining the prognosis of hepatocellular carcinoma [63]. LUZP4 is an mRNA export adaptor that is highly silent in normal tissues other than testes, and is frequently activated in cancers, such as lung cancers, ovarian cancer, melanoma, and multiple myeloma [64,65]. Another study has shown that LRRFIP2 is involved in the selective cleavage of colon cancer and prostate cancer [66]. In addition, LRRFIP2 contains a serin-rich domain that interacts with MyD88 protein, which plays a key role in toll-like receptor 4-mediated signal transduction, and regulates the activity of downstream nuclear factor kappa-light-chain-enhancer of activated B cells (NF-kB) activity [67]. Chromatin dynamics regulate a variety of cell functions, and the destruction of chromatin's homeostasis leads to tumorigenesis and tumor progression. A study has shown that TDRD7 may be used as a histone-binding protein and is crucial in regulating chromatin homeostasis [68]. Currently, few studies on the role of KHDC1L in tumors are available.

Furthermore, the reliability and stability of our model was also analyzed. The results showed that the model accurately distinguished between patients with different prognoses. The ROC curve analysis also showed that our prognostic risk model has good diagnostic capability. The survival analysis and ROC curve analysis were verified in a TCGA test cohort, and the results also support the above conclusion. The univariate and multivariate Cox regression analyses showed that our model independently predicted the prognosis of COAD patients. Subsequently, a nomogram based on our model was constructed to predict the prognosis of COAD patients more intuitively. The HPA database was used to verify the expression of five hub RBPs at the immunohistochemical level. The results showed that the expression of LRRFIP2 and TDRD7 in COAD tissues was significantly higher than in the normal colon tissues, suggesting that LRRFIP2 and TDRD7 may have potential carcinogenic risk, which was consistent with our previous findings. In addition, expression of LUZP4 was not detected in normal colon or COAD tissues, which may be related to the limitations of data included in the databases. In summary, our prognostic prediction model was relatively reliable and could be used to identify COAD patients with poor prognoses, facilitating the early intervention and treatment of COAD patients.

In general, the prognostic model constructed by this study based on five prognosis-related RBPs predicted the prognosis of COAD patients well and helped physicians to make clinical decisions. However, this study still has certain limitations. First, as our prognostic model used data obtained from the TCGA database, our findings will need to be verified in a larger clinical patient cohort in the future. And since TCGA data are from the United States, the conclusions of this study need to be verified in data from other countries and regions to determine whether the results can reflect the situation in other groups. Second, for the specific role of the selected RBPs in COAD, in vitro and animal experiments will be needed in the future to reveal their internal mechanisms.

\section{Conclusion}

This study systemically analyzed the key role and prognostic value of RBPs in COAD by performing univariate and multivariate Cox regression analyses on RBPs that were differentially expressed in tumor and normal tissues. The study ultimately screened five RBPs that were significantly related to COAD prognosis in order to construct a prognostic model. Survival analysis and independent prognosis analysis confirmed that our prognostic model could be used as independent predictor of COAD prognosis. This study helped to further explore the mechanisms of COAD tumorigenesis and progression, and guide the selection of clinical prognostic molecular markers and therapeutic targets for COAD patients.

\section{Acknowledgements}

\section{Author Contributions}

$\mathrm{JH}$ and LX conceived and designed the study; QZ, MY, YW downloaded and organized the TCGA data; XQ and ZL performed data analysis and wrote the paper; $\mathrm{JH}$ and LX critically revised the article for essential intellectual content and administrative support. All authors read and approved the final manuscript.

\section{Availability of data and materials}

The authors declare that the data supporting the findings of this study are available in the TCGA database (https:/ / portal.gdc.cancer.gov/).

\section{Funding}

This work was supported by Beijing University of Chinese Medicine 2020 Basic Research Business Expenses Project (No. 2020-JYB-ZDGG-143-1), National Natural Science Foundation of China General Program (No. 82074545) and Beijing Municipal Natural Science Foundation (No. 7202122). 


\section{Competing Interests}

The authors have declared that no competing interest exists.

\section{References}

1. Siegel RL, Miller KD, Jemal A. Cancer statistics, 2019. CA Cancer J Clin 2019;69:7-34.

2. Banerjee A, Pathak S, Subramanium VD, Dharanivasan G, Murugesan R, Verma RS. Strategies for targeted drug delivery in treatment of colon cancer: current trends and future perspectives. Drug Discov Today 2017;22:1224-1232.

3. Nagtegaal ID, Odze RD, Klimstra D, Paradis V, Rugge M, Schirmacher P, et al. The 2019 WHO classification of tumours of the digestive system. Histopathology 2020;76:182-188.

4. Huang W, Liu Z, Li Y, Liu L, Mai G. Identification of long noncoding RNAs biomarkers for diagnosis and prognosis in patients with colon adenocarcinoma. J Cell Biochem 2019;120:4121-4131.

5. Han X, Kang KA, Piao MJ, Zhen AX, Hyun YJ, Kim HM, et al. Shikonin exerts cytotoxic effects in human colon cancers by inducing apoptotic cell death via the endoplasmic reticulum and mitochondria-mediated pathways. Biomol Ther 2019;27:41-47.

6. Siegel RL, Miller KD, Jemal A. Cancer statistics, 2017. CA Cancer J Clin 2017:67:7-30.

7. Jin L, Li C, Liu T, Wang L. A potential prognostic prediction model of colon adenocarcinoma with recurrence based on prognostic lncRNA signatures. Hum Genomics 2020;14:24

8. Wurth L, Gebauer F. RNA-binding proteins, multifaceted translational regulators in cancer. Biochim Biophys Acta - Gene Regul Mech 2015;1849:881886.

9. Gerstberger S, Hafner M, Tuschl T. A census of human RNA-binding proteins. Nat Rev Genet 2014;15:829-845.

10. Masuda K, Kuwano Y. Diverse roles of RNA-binding proteins in cancer traits and their implications in gastrointestinal cancers. Wiley Interdiscip Rev RNA 2019·10:e1520.

11. Hopkins TG, Mura M, Al-Ashtal HA, Lahr RM, Abd-Latip N, Sweeney K, et al. The RNA-binding protein LARP1 is a post-transcriptional regulator of survival and tumorigenesis in ovarian cancer. Nucleic Acids Res 2016;44:12271246.

12. Wurth L, Papasaikas P, Olmeda D, Bley N, Calvo GT, Guerrero S, et al. UNR/CSDE1 Drives a Post-transcriptional Program to Promote Melanoma Invasion and Metastasis. Cancer Cell 2016;30:694-707.

13. Sánchez-Jiménez C, Ludeña MD, Izquierdo JM. T-cell intracellular antigens function as tumor suppressor genes. Cell Death Dis 2015;6:e1669.

14. Ishii H, Saitoh M, Sakamoto K, Kondo T, Katoh R, Tanaka S, et al. Epithelial splicing regulatory proteins 1 (ESRP1) and 2 (ESRP2) suppress cancer cell motility via different mechanisms. J Biol Chem 2014;289:27386-27399.

15. Li W, Gao LN, Song PP, You CG. Development and validation of a RNA binding protein-associated prognostic model for lung adenocarcinoma. Aging (Albany NY) 2020;12:3558-3573.

16. Li W, Li X, Gao LN, You CG. Integrated Analysis of the Functions and Prognostic Values of RNA Binding Proteins in Lung Squamous Cell Carcinoma. Front Genet 2020;11:185

17. Wang K, Li L, Fu L, Yuan Y, Dai H, Zhu T, et al. Integrated Bioinformatics Analysis the Function of RNA Binding Proteins (RBPs) and Their Prognostic Value in Breast Cancer. Front Pharmacol 2019;10:140

18. Tomczak K, Czerwińska P, Wiznerowicz M. The Cancer Genome Atlas (TCGA): An immeasurable source of knowledge. Wspolczesna Onkol 2015;1A:A68-A77.

19. Ritchie ME, Phipson B, Wu D, Hu Y, Law CW, Shi W, et al. Limma powers differential expression analyses for RNA-sequencing and microarray studies. Nucleic Acids Res 2015;43:e47.

20. Yu G, Wang LG, Han Y, He QY. ClusterProfiler: An R package for comparing biological themes among gene clusters. Omi A J Integr Biol 2012;16:284-287.

21. Harris MA, Clark JI, Ireland A, Lomax J, Ashburner M, Collins R, et al. The gene ontology (GO) project in 2006. Nucleic Acids Res 2006;34:D322-D326.

22. Kanehisa M, Furumichi M, Tanabe M, Sato Y, Morishima K. KEGG: New perspectives on genomes, pathways, diseases and drugs. Nucleic Acids Res 2017:45:D353-D361.

23. Szklarczyk D, Gable AL, Lyon D, Junge A, Wyder S, Huerta-Cepas J, et al. STRING v11: Protein-protein association networks with increased coverage, supporting functional discovery in genome-wide experimental datasets. Nucleic Acids Res 2019:47:D607-D613.

24. Shannon P, Markiel A, Ozier O, Baliga NS, Wang JT, Ramage D, et al. Cytoscape: A software Environment for integrated models of biomolecular interaction networks. Genome Res 2003;13:2498-2504.

25. Bader GD, Hogue CWV. An automated method for finding molecular complexes in large protein interaction networks. BMC Bioinformatics 2003;4:2.

26. Gao J, Aksoy BA, Dogrusoz U, Dresdner G, Gross B, Sumer SO, et al. Integrative analysis of complex cancer genomics and clinical profiles using the cBioPortal. Sci Signal 2013;6:pl1-pl1.
27. Uhlen M, Zhang C, Lee S, Sjöstedt E, Fagerberg L, Bidkhori G, et al. A pathology atlas of the human cancer transcriptome. Science 2017;357: eaan2507.

28. Torres-Fernández LA, Jux B, Bille M, Port Y, Schneider K, Geyer M, et al. The mRNA repressor TRIM71 cooperates with Nonsense-Mediated Decay factors to destabilize the mRNA of CDKN1A/p21. Nucleic Acids Res 2019;47:1186111879 .

29. Glisovic T, Bachorik JL, Yong J, Dreyfuss G. RNA-binding proteins and post-transcriptional gene regulation. FEBS Lett 2008;582:1977-1986.

30. Pereira B, Billaud M, Almeida R. RNA-Binding Proteins in Cancer: Old Players and New Actors. Trends in Cancer 2017:3:506-528.

31. Ye B, Shi J, Kang H, Oyebamiji O, Hill D, Yu H, et al. Advancing Pan-cancer Gene Expression Survial Analysis by Inclusion of Non-coding RNA. RNA Biol 2019:1-8.

32. Hajjari M, Salavaty A. HOTAIR: an oncogenic long non-coding RNA in different cancers. Cancer Biol Med 2015;12:1-9.

33. Ni X, Ding Y, Yuan H, Shao J, Yan Y, Guo R, et al. Long non-coding RNA ZEB1-AS1 promotes colon adenocarcinoma malignant progression via miR-455-3p/PAK2 axis. Cell Prolif 2020;53:e12723.

34. Montanaro L, Treré D, Derenzini M. Changes in ribosome biogenesis may induce cancer by down-regulating the cell tumor suppressor potential. Biochim Biophys Acta - Rev Cancer 2012;1825:101-110.

35. Mironova N, Patutina O, Brenner E, Kurilshikov A, Vlassov V, Zenkova M. The systemic tumor response to RNase A treatment affects the expression of genes involved in maintaining cell malignancy. Oncotarget 2017;8:7879678810 .

36. Goudarzi KM, Lindström MS. Role of ribosomal protein mutations in tumor development (Review). Int J Oncol 2016;48:1313-24.

37. Alam E, Maaliki L, Nasr Z. Ribosomal protein S3 selectively affects colon cancer growth by modulating the levels of p53 and lactate dehydrogenase. Mol Biol Rep 2020;47:6083-6090.

38. Zhang B, Babu KR, Lim CY, Kwok ZH, Li J, Zhou S, et al. A comprehensive expression landscape of RNA-binding proteins (RBPs) across 16 human cancer types. RNA Biol 2020;17:211-226.

39. Silvera D, Formenti SC, Schneider RJ. Translational control in cancer. Nat Rev Cancer 2010;10:254-266.

40. Hayano T, Yanagida M, Yamauchi $\mathrm{Y}$, Shinkawa T, Isobe T, Takahashi N. Proteomic analysis of human Nop56p-associated pre-ribosomal ribonucleoprotein complexes: Possible link between Nop56p and the nucleolar protein treacle responsible for Treacher Collins syndrome. J Biol Chem 2003;278:34309-34319.

41. Turano M, Angrisani A, Rosa MDE, Izzo P, Furia M. Real-time PCR quantification of human DKC1 expression in colorectal cancer. Acta Oncol (Madr) 2008;47:1598-1600.

42. Sieron, P. Hader, C. Hatina, J, Engers, R, Wlazlinski, A, Müller, M, \& Schulz WA. DKC1 overexpression associated with prostate cancer progression. British Journal of Cancer. Br J Cancer 2009;101:1410-1416.

43. Daizumoto K, Yoshimaru T, Matsushita Y, Fukawa T, Uehara H, Ono M, et al. A ddx31/mutant-p53/EGFR axis promotes multistep progression of muscleinvasive bladder cancer. Cancer Res 2018;78:2233-2247.

44. Fukawa T, Ono M, Matsuo T, Uehara H, Miki T, Nakamura Y, et al. DDX31 regulates the p53-HDM2 pathway and rRNA gene transcription through its interaction with NPM1 in renal cell carcinomas. Cancer Res 2012;72:5867-5877.

45. Schütz P, Karlberg T, van den Berg S, Collins R, Lehtiö L, Högbom M, et al. Comparative structural analysis of human DEAD-Box RNA helicases. PLoS One 2010;5:1-11.

46. Wu XL, Yang ZW, He L, Dong P De, Hou MX, Meng XK, et al. RRS1 silencing suppresses colorectal cancer cell proliferation and tumorigenesis by inhibiting G2/M progression and angiogenesis. Oncotarget 2017:8:82968-82980.

47. Ma Y, Yan F, Wei W, Deng J, Li L, Liu L, et al. MicroRNA-598 inhibits the growth and maintenance of gastric cancer stem-like cells by down-regulating RRS1. Cell Cycle 2019;18:2757-2769.

48. Zhang Y, Sun B, Zhao L, Liu Z, Xu Z, Tian Y, et al. Up-regulation of miRNA-148a inhibits proliferation, invasion, and migration while promoting apoptosis of cervical cancer cells by down-regulating RRS1. Biosci Rep 2019:39:BSR20181815.

49. Wang J, Li Z, Zuo C, Xie Q, Li H, Jia J, et al. Knockdown of RRS1 by lentiviral-mediated RNAi promotes apoptosis and suppresses proliferation of human hepatocellular carcinoma cells. Oncol Rep 2017:38:2166-2172.

50. Liu Y, Zhang Y, Chi Q, Wang Z, Sun B. Methyltransferase-like 1 (METTL1) served as a tumor suppressor in colon cancer by activating 7-methyguanosine (m7G) regulated let-7e miRNA/HMGA2 axis. Life Sci 2020;15:117480.

51. Tian QH, Zhang MF, Zeng JS, Luo RG, Wen Y, Chen J, et al. METTL1 overexpression is correlated with poor prognosis and promotes hepatocellular carcinoma via PTEN. J Mol Med 2019;97:1535-45.

52. Pandolfini L, Barbieri I, Bannister AJ, Hendrick A, Andrews B, Webster N, et al. METTL1 Promotes let-7 MicroRNA Processing via m7G Methylation. Mol Cell 2019;74:1278-1290.e9.

53. Wang HL, Chen BB, Cao XG, Wang J, Hu XF, Mu XQ, et al. The clinical significances of the abnormal expressions of Piwil1 and Piwil2 in colonic adenoma and adenocarcinoma. Onco Targets Ther 2015;8:1259-1264.

54. Raeisossadati R, Abbaszadegan MR, Moghbeli M, Tavassoli A, Kihara AH, Forghanifard MM. Aberrant expression of DPPA2 and HIWI genes in colorectal cancer and their impacts on poor prognosis. Tumor Biol 2014;35:5299-5305. 
55. Sun R, Gao CL, Li DH, Li BJ, Ding YH. Expression Status of PIWIL1 as a Prognostic Marker of Colorectal Cancer. Dis Markers 2017;2017:1-7.

56. Jonas K, Calin GA, Pichler M. Rna-binding proteins as important regulators of long non-coding rnas in cancer. Int J Mol Sci 2020;21:2969.

57. Carpenter B, Mckay M, Dundas SR, Lawrie LC, Telfer C, Murray GI. Heterogeneous nuclear ribonucleoprotein $\mathrm{K}$ is over expressed, aberrantly localised and is associated with poor prognosis in colorectal cancer. Br J Cancer 2006;95:921-927.

58. Yang R, Zeng Y, Xu H, Chen Z, Xiang M, Fu Y, et al. Heterogeneous nuclear ribonucleoprotein $\mathrm{K}$ is overexpressed and associated with poor prognosis in gastric cancer. Oncol Rep 2016;36:929-935.

59. Shenoy N, Kessel R, Bhagat TD, Bhattacharyya S, Yu Y, McMahon C, et al. Alterations in the ribosomal machinery in cancer and hematologic disorders. J Hematol Oncol 2012;5:32

60. David CJ, Manley JL. Alternative pre-mRNA splicing regulation in cancer: Pathways and programs unhinged. Genes Dev 2010;24:2343-2364.

61. Meng N, Chen M, Chen D, Chen XH, Wang JZ, Zhu S, et al. Small Protein Hidden in lncRNA LOC90024 promotes "Cancerous" RNA Splicing and Tumorigenesis. Adv Sci 2020;7:1903233.

62. Ding D, Liu J, Midic U, Wu Y, Dong K, Melnick A, et al. TDRD5 binds piRNA precursors and selectively enhances pachytene piRNA processing in mice. Nat Commun 2018;9:127.

63. Wang X, Zhou X, Liu J, Liu Z, Zhang L, Gong Y, et al. Genome-wide investigation of the clinical implications and molecular mechanism of long noncoding RNA LINC00668 and protein-coding genes in hepatocellular carcinoma. Int J Oncol 2019;55:860-878.

64. Türeci Ö, Sahin U, Koslowski M, Buss B, Bell C, Ballweber P, et al. A novel tumour associated leucine zipper protein targeting to sites of gene transcription and splicing. Oncogene 2002;21:3879-3988.

65. Viphakone N, Cumberbatch MG, Livingstone MJ, Heath PR, Dickman MJ, Catto JW, et al. Luzp4 defines a new mRNA export pathway in cancer cells. Nucleic Acids Res 2015;43:2353-2366.

66. Thorsen K, Sørensen KD, Brems-Eskildsen A, Modin C, Gaustadnes M, Hein AMK, et al. Alternative splicing in colon, bladder, and prostate cancer identified by exon array analysis. Mol Cell Proteomics 2008;7:1214-1224.

67. Gunawardena HP, Huang Y, Kenjale R, Wang H, Xie L, Chen X. Unambiguous characterization of site-specific phosphorylation of leucine-rich repeat fli-i-interacting protein 2 (LRRFIP2) in toll-like receptor 4 (TLR4)-mediated signaling. J Biol Chem 2011;286:10897-10910.

68. Bua DJ, Kuo AJ, Cheung P, Liu CL, Migliori V, Espejo A, et al. Epigenome microarray platform for proteome-wide dissection of chromatin-signaling networks. PLoS One 2009:4:e6789. 